\title{
Wide band observations of the X-ray burster GS 1826-238
}

\author{
M. Cocchi ${ }^{1}$, R. Farinelli ${ }^{2}$, A. Paizis ${ }^{3}$, and L. Titarchuk ${ }^{2,4,5,6}$ \\ 1 INAF, Istituto di Astrofisica Spaziale e Fisica Cosmica sez. di Roma, via Fosso del Cavaliere, 100, 00133 Roma, Italy \\ e-mail: Massimo.Cocchi@iasf-roma.inaf.it \\ 2 Dipartimento di Fisica, Universitá di Ferrara, Italy \\ 3 INAF, Istituto di Astrofisica Spaziale e Fisica Cosmica, sez. di Milano, Italy \\ ${ }^{4}$ Center for Earth Observing and Space Research, George Mason University, Fairfax, VA, USA \\ 5 High Energy Space Environment Branch, US Naval Reserch Laboratory, Washington, DC, USA \\ ${ }^{6}$ Gravitational Astrophysics Laboratory, NASA Goddard Space Flight Center, Greenbelt, MD, USA
}

Received 30 June 2009 / Accepted 19 November 2009

ABSTRACT

\begin{abstract}
Context. GS 1826-238 is a well-studied X-ray bursting neutron star in a low mass binary system. Thermal Comptonisation by a hot electron cloud $\left(k T_{\mathrm{e}} \sim 20 \mathrm{keV}\right)$ is a widely accepted mechanism accounting for its high energy emission, while the nature of most of its soft X-ray output is not completely understood. A further low energy component is typically needed to model the observed spectra: pure blackbody and Comptonisation-modified blackbody radiation by a lower temperature (a few keV) electron plasma were suggested to explain the low energy data.

Aims. In order to better characterise the nature of the low energy emission and the bolometric output of the source, the steady emission of GS 1826-238 is studied by means of sensitive, broad band (X to soft Gamma-rays) measurements obtained by the INTEGRAL observatory.

Methods. In this data analysis, the newly developed, up-to-date Comptonisation model COMPTB is applied for the first time to study effectively the low-hard state variability of a low-luminosity neutron star in a low-mass X-ray binary system. Three joint ISGRI/JEM$X$ data sets (two from observations performed in 2003 and one from 2006) were analysed.

Results. We confirm that the 3-200 keV emission of GS 1826-238 is characterised by Comptonisation of soft seed photons by a hot electron plasma. A single spectral component is sufficient to model the observed spectra. At lower energies, no direct blackbody emission is observed and there is no need to postulate a low temperature Compton region. Compared to the 2003 measurements, the plasma temperature decreased from $\sim 20$ to $\sim 14 \mathrm{keV}$ in 2006, together with the seed photons temperature. The source intensity was also found to be $\sim 30 \%$ lower in 2006 , whilst the average recurrence frequency of the X-ray bursts significantly increased. Possible explanations for this apparent deviation from the typical limit-cycle behaviour of this burster are discussed.
\end{abstract}

Key words. X-rays: general - X-rays: binaries - X-rays: individuals: GS 1826-238

\section{Introduction}

The persistent X-ray source GS 1826-238 was discovered during a manoeuvre of the Ginga satellite in 1988 (Makino et al. 1988). Early band-limited observations of this object were performed by TTM (in 't Zand 1992), and ROSAT (Barret et al. 1995) at low energies and by CGRO-OSSE (Strickman et al. 1996), in the hard X-rays. An optical counterpart in a low inclination system and with a likely orbital period of $2.1 \mathrm{~h}$ was reported (Homer et al. 1998). The detection of type I X-ray bursts (Ubertini et al. 1997) by BeppoSAX ultimately established that the source is a neutron star (NS) in a low mass binary system (LMXB). X-ray bursts were found to recur every few hours in a quasi-periodical fashion (Ubertini et al. 1999). What makes this burster unique among its class is the remarkable stability of the recurrence over a time-scale of several years (Cocchi et al. 2001). Bursting behaviour is clearly related to the persistent emission (Cornelisse et al. 2003), the event frequency increasing with the measured X-ray flux. RXTE data permitted more accurate studies of the burst recurrence properties (see e.g. Galloway et al. 2004), and a possible $611 \mathrm{~Hz}$ oscillation in the burst decay was also reported (Thompson et al. 2005). The lack of evidence for photospheric radius expansion during the bursts (in 't Zand et al. 1999) and general considerations on the optical counterpart (LMXB, short orbital period) limit the value of the distance to the source in the $\sim 4-9 \mathrm{kpc}$ range (Barret et al. 2000).

The history of sensitive, wide band measurements of the steady emission of GS 1826-238 includes results from BeppoSAX (in 't Zand et al. 1999; Del Sordo et al. 1999), RXTE (Barret et al. 2000), RXTE plus Chandra (Thompson et al. 2005), and RXTE plus XMM (Thompson et al. 2008). Since the BeppoSAX era, the source is always detected in a hard (low) spectral state, its high energy emission extending well in excess of $100 \mathrm{keV}$. A simple, empirical, exponentially-cutoff power law model with $E_{\mathrm{c}} \sim 50 \mathrm{keV}$ yields acceptable fits to the high energy data (e.g. Del Sordo et al. 1999) but whenever more physical models are considered, the spectra are generally well described by a Comptonisation scenario, where soft seed photons are up-scattered to high energies by a hot electron plasma $\left(k T_{\mathrm{e}} \sim 20 \mathrm{keV}\right)$. Along with Comptonisation, either a low energy blackbody (BB) spectral component (in 't Zand et al. 1999) or a low-temperature Compton component (Thompson et al. 2005) are included to fit the data. The detection of a $\sim 6 \mathrm{keV}$ iron line was reported by Barret et al. (2000), who also included reflection 
Table 1. Observation $\log$.

\begin{tabular}{|c|c|c|c|}
\hline INTEGRAL revolution & $061-064$ & 119-122 & 495 \\
\hline obs. year & 2003 & 2003 & 2006 \\
\hline obs. day & Apr. $15-23$ & Oct. $06-15$ & Nov. 2 \\
\hline JEM-X live time (ks) & 21.9 & 32.8 & 49.9 \\
\hline ISGRI live time (ks) & 134.4 & 206.5 & 50.3 \\
\hline \# of bursts (JEM-X) & 3 & 5 & 4 \\
\hline average burst delay (h) & 3.79 & 3.77 & 3.40 \\
\hline ASM intensity $(\mathrm{mCrab})^{a}$ & $37 \pm 7$ & $40 \pm 8$ & $25 \pm 4$ \\
\hline
\end{tabular}

a Average during the INTEGRAL observations, $2-10 \mathrm{keV}$.

to fit their data. Evidence for transient soft blackbody emission, possibly related to irregularities in the time behaviour of the X-ray bursts, has recently reported by Thompson et al. (2008).

We present wide band spectral analysis of GS 1826-238 performed by INTEGRAL data. In the next section, observation and data analysis details will be given. In Sect. 3 we present and discuss our results along with our best fit models, while the implications of these results and their relation to the current knowledge of this peculiar X-ray source are debated in Sect. 4.

\section{Observations and data analysis}

The public database ${ }^{1}$ of INTEGRAL (Winkler et al. 2003) includes a big set of useful pointings of GS 1826-238, thanks to the observing policy of the telescope which includes periodic scans of the Galactic plane and the Galactic bulge region (GS 1826-238 is only $\sim 10$ degrees from SgrA*).

Detailed analysis of the whole INTEGRAL GS 1826-238 public database, focused on the source long term monitoring, is to be presented elsewhere (Cocchi et al., in prep.). The present work, focused on wide band spectroscopy of the persistent emission, relies on data obtained by the imaging detectors JEM-X (Lund et al. 2003) and IBIS/ISGRI (Lebrun et al. 2003, hereafter ISGRI), which operate in the $2-30 \mathrm{keV}$ and $17-300 \mathrm{keV}$ passbands, respectively.

Being the source variable, though always observed in a low-hard spectral state, homogeneous data sets are needed: this means that one has to choose simultaneuos JEM-X+ISGRI pointings of sufficient length to get good statistics and as close in time as possible. Due to the different field of view of the two detectors and the typical dithering observing pattern of INTEGRAL, JEM-X live time is considerably less than the ISGRI one.

Taking into account the above limitations, we selected three data sets, the first one belonging to the satellite revolutions 061-064 (visibility window of Spring 2003), the second one to the revolutions 119-122 (Autumn 2003), and the third one to revolution 495 (Autumn 2006). Together, the three sets account for $104.6 \mathrm{ks}$ and $391.2 \mathrm{ks}$ live time (JEM-X and ISGRI, respectively). Analysis of RXTE/ASM monitoring data of GS 1826-238 obtained in the same epochs of our INTEGRAL observations indicate that the $2-10 \mathrm{keV}$ source intensity significantly varied from 2003 to 2006, being 40 mCrab in 2003 and only 25 mCrab in Autumn 2006 (see Table 1). This is confirmed with higher accuracy and on a wider energy band by the present measurements (see Sect. 3).

In order to avoid possible low-energy spectral contamination from the X-ray burst emission, the time intervals of the observed bursts, each event typically lasting $100 \mathrm{~s}$ in JEM-X data,

${ }^{1}$ http://isdc.unige.ch/index.cgi?Data+info were removed from the good time of the data sets. Details about the analysed data sets such as live time, number of bursts detected and inferred average burst periodicities, are summarised in Table 1.

JEM-X data were reduced and extracted by the standard INTEGRAL off-line analysis (OSA) pipeline ${ }^{2}$ release 7.0. Presence of systematic effects at the lowest energies suggested a discarding of the spectral bins in the $2-3 \mathrm{keV}$ range. ISGRI data were reduced by the standard OSA 6.0 pipeline, while spectral extraction was performed by a dedicated software based on cross correlation, which is freely available to the community ${ }^{3}$. The effectiveness and the limitations of the software in terms of calibration and systematics have already been discussed elsewhere (Segreto \& Ferrigno 2006; Mineo et al. 2006). Finally, the joint JEM-X/ISGRI spectra were analysed and best-fitted by the standard software tools of the XSPEC package ${ }^{4}$, version 12 .

Due to cross calibration uncertainties between the two instruments, whose effects are expected to vary with the observation epoch ${ }^{2}$, a constant normalisation factor was included in each fit. We kept ISGRI as a reference, allowing the JEM-X constant to vary. No systematics, accounting for possible instrumental miscalibration, had to be included for both JEM-X and ISGRI.

Our data cannot constrain the low energy absorption along the line of sight, so the $N_{\mathrm{H}}$ value, modeled by Wisconsin absorber in XSPEC, was kept fixed to $3 \times 10^{21} \mathrm{H} \mathrm{cm}^{-2}$, which is the best determined value from recent joint XMM-RXTE measurements (Thompson et al. 2008). Slightly different values, $5 \times 10^{21} \mathrm{H} \mathrm{cm}^{-2}$ and $1.5 \times 10^{21} \mathrm{H} \mathrm{cm}^{-2}$ were determined respectively by previous ROSAT and BeppoSAX measurements (Barret et al. 1995; in 't Zand et al. 1999). All the values are of the same order of magnitude of the interpolated one (Dickey \& Lockman 1990) that can be derived by $\mathrm{NH}$, a widely used ftool ${ }^{5}$. We already verified that these slight differences in the $N_{\mathrm{H}}$ value do not affect the parameters of our best fits and the possible undetection of further soft components (see Sect. 3).

\section{Spectroscopic results}

Our wide band INTEGRAL spectra of GS 1826-238 can be satisfactorily fit in a first approximation by a simple, empirical, exponentially cutoff power-law model, XSPEC 's CUTOFFPL (see Table 2). The power-law photon index is $\sim 1.6$ and the cutoff energy is $\sim 50 \mathrm{keV}$ in all the observations. Such values are in good agreement with all the previous results based on this simple model, thus suggesting that the overall spectral shape (and so the spectral state) of the source is almost constant with time on a time scale of several years.

From a less empirical, more physical point of view, a single high energy cutoff power law is a clear indication of a single Compton region at work. This is (at least partially) in agreement with most of the previous results, but not with those obtained by Thompson et al. (2005) (see also Thompson et al. 2008), who included a second, distinct Compton component with a different geometry and electron temperature to fit their joint Chandra /RXTE data: in our case, as CUTOFFPL is an acceptable model, there is a clear indication that the data themselves

\footnotetext{
2 http://isdc.unige.ch/index.cgi?Soft+info

3 http://www.ifc.inaf.it/ ferrigno/INTEGRALsoftware. html

4 http://heasarc.nasa.gov/docs/xanadu/xspec/index.html

5 http://heasarc.nasa.gov/docs/software/ftools/

ftools_menu.html
} 
Table 2. Spectroscopic results.

\begin{tabular}{|c|c|c|c|}
\hline Data set (revolution) & 061-064 & 1119-122 & 495 \\
\hline \multicolumn{4}{|c|}{$\mathrm{WABS}^{*} \mathrm{COMPTB}^{a}$} \\
\hline$k T_{\mathrm{s}}(\mathrm{keV})$ & $1.06_{-0.06}^{+0.07}$ & $0.95 \pm 0.05$ & $0.45_{-0.14}^{+0.09}$ \\
\hline$\alpha$ index & $1.05_{-0.03}^{+0.04}$ & $1.03 \pm 0.03$ & $0.85_{-0.01}^{+0.02}$ \\
\hline$k T_{\mathrm{e}}(\mathrm{keV})$ & $20.1_{-1.2}^{+1.7}$ & $19.4_{-0.9}^{+1.0}$ & $13.5 \pm 0.8$ \\
\hline const. $^{b}$ & $0.671_{-0.032}^{+0.028}$ & $0.732_{-0.025}^{+0.027}$ & $0.932_{-0.037}^{+0.029}$ \\
\hline red. $\chi^{2}$ (d.o.f.) & $1.076(360)$ & $1.092(361)$ & $1.094(344)$ \\
\hline flux $2-20 \mathrm{keV}^{c}$ & $1.11 \times 10^{-9}$ & $1.34 \times 10^{-9}$ & $1.02 \times 10^{-9}$ \\
\hline flux $20-200 \mathrm{keV}$ & $1.23 \times 10^{-9}$ & $1.34 \times 10^{-9}$ & $0.80 \times 10^{-9}$ \\
\hline flux $2-200 \mathrm{keV}$ & $2.34 \times 10^{-9}$ & $2.68 \times 10^{-9}$ & $1.82 \times 10^{-9}$ \\
\hline luminosity ${ }^{d}\left(\mathrm{erg} \mathrm{s}^{-1}\right)$ & $1.76 \times 10^{37}$ & $1.92 \times 10^{37}$ & $1.42 \times 10^{37}$ \\
\hline$\tau^{e}$ & 3.4 & 3.5 & 5.3 \\
\hline$C A F^{d}$ & 3.8 & 3.9 & 11.5 \\
\hline \multicolumn{4}{|c|}{ WABS $*$ COMPTT } \\
\hline$k T_{0}(\mathrm{keV})$ & $1.02 \pm 0.06$ & $0.91 \pm 0.05$ & $0.33 \pm 0.04$ \\
\hline$k T_{\mathrm{e}}(\mathrm{keV})$ & $21.5_{-1.7}^{+2.2}$ & $20.0_{-1.0}^{+1.2}$ & $14.6_{-1.1}^{+1.3}$ \\
\hline$\tau^{f}$ & $3.81_{-0.36}^{+0.33}$ & $4.12_{-0.24}^{+0.23}$ & $5.55_{-0.35}^{+0.33}$ \\
\hline const. & $0.680_{-0.029}^{+0.031}$ & $0.756_{-0.025}^{+0.026}$ & $0.900_{-0.041}^{+0.043}$ \\
\hline red. $\chi^{2}$ (d.o.f.) & $1.064(360)$ & $1.081(361)$ & $1.070(344)$ \\
\hline flux $2-200 \mathrm{keV}$ & $2.34 \times 10^{-9}$ & $2.68 \times 10^{-9}$ & $1.85 \times 10^{-9}$ \\
\hline \multicolumn{4}{|c|}{ WABS ${ }^{*}$ CUTOFFPL } \\
\hline$E_{\text {cut }}(\mathrm{keV})$ & $51.9_{-3.5}^{+4.0}$ & $55.9_{-2.9}^{+3.2}$ & $49.0_{-5.1}^{+6.1}$ \\
\hline & $1.57 \pm 0.05$ & $1.62 \pm 0.03$ & $1.62 \pm 0.04$ \\
\hline const. & $0.784_{-0.034}^{+0.035}$ & $0.829_{-0.027}^{+0.028}$ & $0.825_{-0.037}^{+0.038}$ \\
\hline red. $\chi^{2}$ (d.o.f.) & $1.022(361)$ & $1.065(362)$ & $1.001(345)$ \\
\hline flux $2-200 \mathrm{keV}$ & $2.46 \times 10^{-9}$ & $2.83 \times 10^{-9}$ & $1.91 \times 10^{-9}$ \\
\hline
\end{tabular}

${ }^{a}$ Name of the Xspec model. $n_{\mathrm{H}}$ fixed to $3.0 \times 10^{21} \mathrm{H} \mathrm{cm}^{-2}$ in all fits All errors are $90 \%$ probability. ${ }^{b}$ JEM-X/ISGRI normalisation ratio (see Sect. 2). ${ }^{c}$ All fluxes in erg cm${ }^{-2} \mathrm{~s}^{-1} .{ }^{d}$ Unabsorbed, $0.1-200 \mathrm{keV}$, assumed distance of $7 \mathrm{kpc} .{ }^{e}$ Derived parameter (see Sect. 3). ${ }^{f}$ Assumed spherical geometry.

do not require the use of too many free parameters to explain the observed emission from GS 1826-238.

Taking all this into account, we tried to fit the data with a single thermal Compton component. The recently developed thermal plus bulk Comptonisation model (Farinelli et al. 2008), COMPTB, included in the standard XSPEC distribution ${ }^{6}$, is presently the most up-to-date Comptonisation model in a diffusion regime (such approximation holds well for NS-LMXBs). COMPTB is a general model including, in a self-consistent way, the seed photons emission together with the Comptonised emission, whether the latter is either purely thermal (as in the well-known model COMPTT), or mixed dynamical (bulk Comptonisation) plus thermal. As a detailed description of the model is given in Farinelli et al. (2008), we here only point out that the free parameters of COMPTB are the blackbody seed photon and the electron temperatures $\left(k T_{\mathrm{s}}\right.$ and $k T_{\mathrm{e}}$, respectively), the Comptonisation index $\alpha$, the bulk parameter $\delta$, the covering factor $\log (A)$ and the normalisation.

As already mentioned above, the spectrum of GS 1826238 is well described by a simple function (CUTOFFPL), which mimics unsaturated Comptonisation for static plasma and no blackbody-like peak is observed at low energies: the case of the seed blackbody radiation dominated by the Compton emission is modeled by СОMPTB with a high value of the covering factor, i.e. $A \gg 1$. Moreover, GS $1826-238$ being a low luminosity (a

\footnotetext{
${ }^{6}$ http://heasarc.nasa.gov/docs/xanadu/xspec/newmodels. html
}

few \% Eddington) NS-LMXB in a low (hard) state, the detection of a bulk emission is unlikely (Paizis et al. 2006, see also Sect. 4.1). These two particular cases are well reproduced in our fits: when allowed to vary, both the $\log (A)$ and the $\delta$ parameters are eventually pegged by the fitting procedure to their hard limits corresponding to total coverage $(\log (A)=8)$ and no bulk emission $(\delta=0)$, thus reproducing a simple thermal Comptonisation spectrum without direct emergence of the underlying seed photons. We then fixed $\delta$ and $\log (A)$ to the above limit values in all the fits, leaving only $k T_{\mathrm{s}}, k T_{\mathrm{e}}$ and $\alpha$ as variable parameters. In this particular case, the dimension of the free-parameter space is identical to that of the well consolidated and widely adopted COMPTT model (Titarchuk 1994), and the two models are substantially equivalent.

The results of our СОMPTB fits are reported in Table 2 for each of the three different observation periods. Spectra and residuals to the best fits are also shown in Fig. 1 for two selected observations. A single COMPTB component with $k T_{\mathrm{e}}$ in the range $15-20 \mathrm{keV}$ effectively describes the shape of the spectra. Though the source persisted in its low-hard state, significantly different best fit values were obtained for both electron plasma and soft seed photon temperatures in the 2003 and 2006 observations. The 2006 data set required lower temperatures and a lower value of the index $\alpha$, indicating an increased efficiency of the Compton upscattering. The $2-200 \mathrm{keV}$ intensity also dropped by $\sim 30 \%$ with respect to 2003 . We carefully verified that the different values of the main spectral parameters in 2006 are not biased by a probable long term variation of the JEM-X/ISGRI normalisation constant: in fact consistent $k T_{\mathrm{e}}$ values are obtained even when forcing the 2003 cross-calibration in the 2006 data set, and vice versa. Moreover, considering only ISGRI data for the fits does not alter the main parameter values. Finally, the $R X T E$ /ASM data independently confirm in a narrower passband the drop in the flux (see Table 1).

Best-fit excesses above $\sim 75 \mathrm{keV}$ are observed in the 2006 data set (see lower panel of Fig. 1) and partly in the Autumn 2003 one. But they are not detected in Spring 2003 and moreover their significance is poor. So we regard such residuals as random fluctuations.

Interesting additional physical information can be extracted from the best-fit parameters of СОМРТВ; for example, the cloud optical depth $\tau$ can be derived by the Eqs. (17)-(24) in Titarchuk $\&$ Lyubarskij (1995). The $\tau$ values, assuming a spherical geometry, are included in Table 2. For a direct comparison, the results obtained replacing СОМРТB with the more popular model COMPTT (spherical geometry assumed) are also reported in Table 2. As expected, the main COMPTT parameter values are in good agreement with the COMPTB ones.

Moreover, the order of magnitude of the radius of the (assumed) spherical region emitting the blackbody seed photons can be estimated: as the normalisation in COMPTB is defined as $C_{\mathrm{N}}=L_{39} / D_{10}^{2}$, where $L_{39}$ is the seed photon spectrum luminosity (in units of $10^{39} \mathrm{erg} \mathrm{s}^{-1}$ ) and $D_{10}$ is the source distance (in units of $10 \mathrm{kpc}$ ), one can derive applying the Stefan-Boltzmann law the emitting region radius as

$R_{\mathrm{km}}=0.88 \times 10^{2} \frac{C_{\mathrm{N}}^{1 / 2} D_{10}}{k T_{\mathrm{s}}^{2}}$.

We obtain for the three observational data sets the values $R_{\mathrm{km}}=$ 6,8 and $24 \mathrm{~km}$, respectively (a source distance of $7 \mathrm{kpc}$ is assumed).

The X-ray energy amplification caused by the Compton scattering can also be determined by computing the Compton 


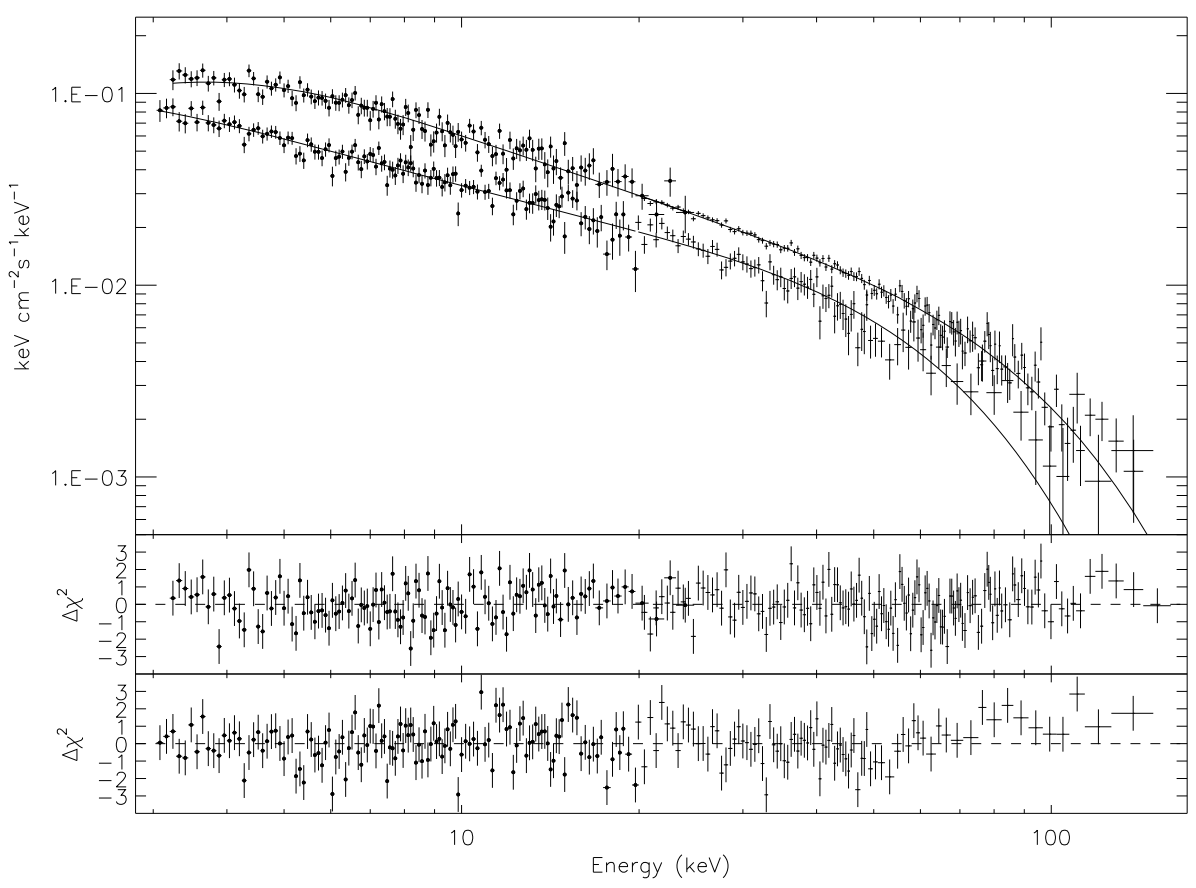

Fig. 1. The INTEGRAL spectrum of GS $1826-238$ as observed in Autumn 2003 (brighter spectrum) and Autumn 2006. Simple crosses and dotted crosses are used for ISGRI and JEM-X, respectively. Residuals to the best СОMPTB fits are shown in the lower panels for the 2003 (above) and 2006 data (below).
Amplification Factor $(C A F)$, defined as the ratio of the Comptonised bolometric energy flux to that of the blackbody seed radiation. The obtained values of the parameter for each observation of GS 1826-238 are also listed in Table 2. Increased Compton efficiency, as already testified by the correspondingly decreasing value of the $\alpha$ index, is observed in 2006.

\section{Discussion}

\subsection{GS 1826-238 in a unifying framework}

We applied a recently developed model for Compton upscattering, СОМРТВ, to study the low-hard state variability of GS 1826-238. As Paizis et al. (2006) demonstrated, general Comptonisation models including bulk emission are very effective in modeling the variety of NS-LMXBs spectral states and the state transitions in individual sources. This is obtained, in a natural way, by smooth changes in the relative importance of bulk over thermal Comptonisation. In this scenario having the interplay of bulk and thermal Compton emission as unifying parameter, a low-hard state is expected to originate at low accretion rates, where the coronal Compton cooling is far from being efficient, thus leaving out a hot screen which extends radially and spherically, thus shielding the NS surface and allowing the direct accretion disk emission to be cooler. Bulk motion Comptonisation can still occur in this case, as the NS gravity is not balanced by the radiation pressure, but the effect is expected to be weak and probably washed in the hot screen with negligible effects for a distant observer.

The present results confirm the above picture for the case of GS 1826-238, as pure thermal upscattering completely accounts for the source emission. The relative extension of the Compton cloud determines whether it is possible to observe a fraction of the seed photons' population originating from the NS surface and/or from the inner accretion disk. In our spectra the soft blackbody seed photons are not directly detected, given the inferred $\log A \gg 1$, and no further soft components are needed to fit the data.

\subsection{Wide band spectroscopy: extra spectral components and variability}

Undetection of additional soft components in the INTEGRAL data is in agreement with the results obtained by Strickman et al. (1996) and Barret et al. (2000) for the same source, but not with those of in't Zand et al. (1999) and Del Sordo et al. (1999), who proposed an extra blackbody $\left(k T_{\mathrm{bb}}\right.$ of $\sim 3 \mathrm{keV}$ and $\sim 1 \mathrm{keV}$, respectively), in addition to a thermally Comptonised component (or a Compton-like, in the case of Del Sordo et al. 1999), to fit the BeppoSAX data. Our upper limits for a further blackbody-like component were calculated, assuming a typical colour temperature of $1 \mathrm{keV}$ and a distance of $7 \mathrm{kpc}$. We obtained blackbody emitting regions with radii $R<1.0 \mathrm{~km}$ and $R<0.7 \mathrm{~km}$ for the 2003 and 2006 data sets, respectively ( $90 \%$ confidence).

Applying a PEXRAV model to our data as in Barret et al. (2000) does not improve the fits. The simple cutoff power law is reproduced, but the reflection parameter is pegged to zero. This is expected, as CUTOFFPL always yields acceptable fits and there is no need for further hypotheses.

For GS 1826-238, Thompson et al. (2005) and (2008) proposed a model with two distinct Comptonising regions, one with disk geometry and a high $(\sim 20 \mathrm{keV})$ temperature, the other of spherical shape and a lower $(\sim 6 \mathrm{keV})$ temperature. The two separate regions had different opacities and seed photons populations. This two-cloud model actually fits our data, with values very close to those obtained by the above authors, but this is not very surprising because it has many free parameters. As a matter of fact, our data do not need too many free parameters because a simple cutoff powerlaw provides a satisfactory fit. Moreover, a single powerlaw is an indication of a single Comptonising region at work. In Thompson et al. (2005), much simpler models (e.g. CUTOFFPL) yielded acceptable fits to their data as well; interestingly, all the fits had very low $(\sim 0.7-0.8)$ reduced chisquared, probably indicating systematic effects at work, such as error overestimation or missed rebinning.

It must be stressed that the presence of a second, softer, spectral component cannot be completely ruled out by the present 


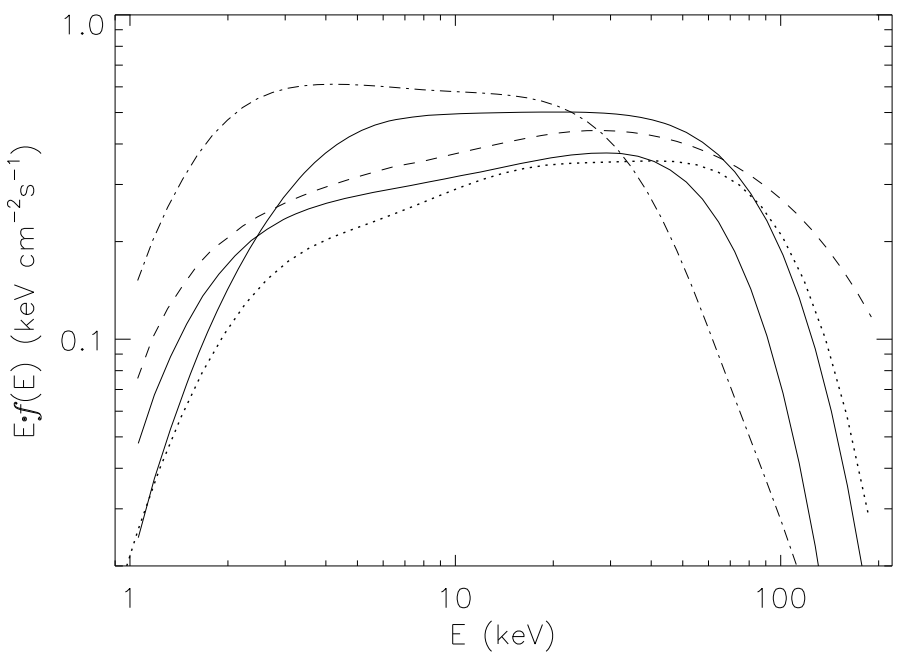

Fig. 2. Comparison of wide band spectra of GS 1826-238 obtained by different observations: our INT EGRAL spectra, as in Fig. 1 (simple lines); RXTE (Barret et al. 2000, dashed line); Chandra+RXTE (Thompson et al. 2005, dash-dotted); BeppoSAX (in 't Zand et al. 1999, dots). The Fe lines, even when detected, are not included.

INTEGRAL data. But its actual need should be justified by the overall X-ray spectral shape obtained including the lowest energy range $(E<2-3 \mathrm{keV})$, which is not covered by JEM-X. This is likely, as both the BeppoSAX and Chandra already published spectra start well below $1 \mathrm{keV}$. Nevertheless, both the previously reported $\sim 3 \mathrm{keV}$ blackbody-like component (in 't Zand et al. 1999) and the $\sim 5 \mathrm{keV}$ Comptonised emission (Thompson et al. 2005) are well inside the INTEGRAL passband and should be unambiguously constrained. This is not the case.

The present analysis shows clear evidence for intensity and spectral variability of GS 1826-238 (even though almost unexpected, given the peculiar long time-scale regularity of its bursting behaviour, which is suggestive of a stable, smooth accretion). In particular, significantly lower plasma temperatures and 2-200 keV luminosity are observed in 2006 compared with 2003.

A comparison of our results with previously reported wide band observations of GS 1826-238 is shown in Fig. 2. While at least approximately the canonical low-state spectral hardening with source dropping luminosity is well reproduced by our 2003 data, the unusually low electron plasma temperature of 2006 coupled with relatively low luminosity seems to contradict this consolidated scenario. Together with the probably erratic presence of blackbody-like emission at lower energies (see also below), the overall picture of GS 1826-238 is suggestive of the emergence of possible sub-states within its low-hard state. In such a spectral state, different observational characteristics could be attributed to differences in the configuration of the accreting system, with the size and geometry of the Compton cloud likely playing a pivotal role.

\subsection{X-ray bursts timing irregularities}

Taking into account the observed decrease of the X-ray bursts' wait times together with lower measured intensity (see Table 1), our November 2006 data show a clear deviation from the typical limit-cycle X-ray bursting behaviour of GS 1826-238. Burst wait times $\Delta T$ are generally anticorrelated with very good approximation to the source intensity $F_{X}$ by the simple formula $\Delta T \propto F_{\mathrm{X}}^{-1.05}$ (see Galloway et al. 2004). If one were to consider the measured $2-200 \mathrm{keV}$ flux as an indication of the actual accretion rate as well as the burst fuel accumulation rate, a deviation of $\sim 35 \%$ from the limit-cycle rule would be obtained with respect to e.g. the 2003 INTEGRAL data. Similar anomalies were already observed in the April 2003 RXTE data (Thompson et al. 2008). These results indicate that spectral measurements which do not include the very soft X-rays are unreliable tools to extrapolate the bolometric luminosity, which can be widely underestimated. In fact Thompson et al. (2008) reported of a simultaneous XMM-observed transient blackbody-like spectral component. This very soft (a few tenths of keV) component accounted for the missing flux, allowing for a better estimate of the bolometric luminosity and so of the true accretion rate. Our INTEGRAL results can also be explained in the same natural way, leaving out early burst ignition and/or anisotropy considerations. But a further very soft component similar to the one reported by Thompson et al. (2008) would then remain undetected, being outside of the JEM-X passband. Nevertheless, our 2006 data show a significant decrease of both the Compton cloud electron and the seed photon temperatures, which is possibly related to an overall bolometric spectral variation of GS 1826-238.

The above reported erratic variability of the source, in terms of both transient soft components and/or Comptonisation parameters, deserves to be studied in detail to be better understood and implies the need for further accurate wide band (from very soft X-rays up to soft Gamma-rays) monitoring programs of GS 1826-238 and other similar X-ray bursters.

Acknowledgements. This work has been supported by the grant from the INAF PRIN 2007 Bulk motion Comptonization models in X-ray Binaries: from phenomenology to physics, PI M. Cocchi. A.P. acknowledges the Italian Space Agency financial and programmatic support via contract I/008/07/0. The authors thank C. Ferrigno and A. Segreto for useful suggestions and tips.

\section{References}

Barret, D., Motch, C., \& Pietsch, W. 1995, A\&A, 305, 526 Barret, D., Olive, J. F., Boirin, L., et al. 2000, ApJ, 533, 329 Cocchi, M., Bazzano, A., Natalucci, L., et al. 2001, Adv. Space Res., 28, 375 Cornelisse, R., in 't Zand, J. J. M., Verbunt, F., et al. 2003, A\&A, 405, 1033 Del Sordo, S., Frontera, F., Pian, E., et al. 1999, Astrophys. Lett. Comm., 38, 125

Dickey, J., \& Lockman, F. 1990, ARA\&A, 28, 215

Farinelli, R., Titarchuk, L., Paizis, A., \& Frontera, F. 2008, ApJ, 680, 602 Galloway, D. K., Cummings, A., Kuulkers, E., et al. 2004, ApJ, 601, 466 Homer, L., Charles, P. A., \& O'Donoghue, D. 1998, MNRAS, 298, 497

Lebrun, F., Leray, J. P., Lavocat, P., et al. 2003, A\&A, 411, L141

Lund, N., Budtz-Jørgensen, C., Westergaard, N. J., et al. 2003, A\&A, 411, L231 Makino, R., et al. 1988, IAU Circ., 4653

Mineo, T., Ferrigno, C., Foschini, L., et al. 2006, A\&A, 450, 617

Paizis, A., Farinelli, R., Titarchuk, L., et al. 2006, A\&A, 459, 187

Segreto, A., \& Ferrigno, C. 2006, in Proc. of the 6th INTEGRAL Workshop The Obscured Universe, Moscow on 2-8 July 2006, ed. S. Grebenev, R. Sunyaev, \& C. Winkler, 633

Strickman, M., Skibo, J., Purcell, W., Barret, D., \& Motch, C. 1996, A\&AS, 120, 217

Thompson, T. W. J., Rothschild, R. E., Tomsick, J. A., \& Marshall, H. L. 2005, ApJ, 634, 1261

Thompson, T. W. J., Galloway, D. K., Rothschild, R. E., \& Homer, L. 2008, ApJ, 681, 506

Titarchuk, L. 1994, ApJ, 434, 570

Titarchuk, L., \& Lyubarskij, Y. 1995, ApJ, 450, 876

Ubertini, P., Bazzano, A., Cocchi, M., et al. 1997, IAU circ. 6611

Ubertini, P., Bazzano, A., Cocchi, M., et al. 1999, ApJ, 514, L27

Winkler, C., Courvoisier, T. J.-L., Di Cocco, G., et al. 2003, A\&A, 411, L1

in 't Zand, J. J. M. 1992, Ph.D. Thesis, University of Utrecht

in 't Zand, J. J. M., Heise, J., Kuulkers, E., et al. 1999, A\&A, 347, 891 